\title{
Global CKM Fits of the CKM Matrix with the Scan Method
}

\author{
Gerald Eigen, ${ }^{* \dagger}$ \\ University of Bergen \\ E-mail: gerald.eigeneift.uib.no
}

\section{Gregory Dubois-Felsmann, David G. Hitlin, Frank C. Porter}

\section{Caltech}

E-mail: gpdfeslac.stanford.edu, hitlinecaltech.edu, fcpecaltech.edu

\begin{abstract}
We present updated global fits of the CKM matrix with the Scan Method, now including the measurements of $B_{s}^{0} \rightarrow \mu^{+} \mu^{-}$and $B_{d}^{0} \rightarrow \mu^{+} \mu^{-}$branching fractions in the baseline fit. We explore the theory parameter space by scanning over three of the ten observables that have significant theory uncertainties. Furthermore, we explore the impact of a model that assumes new physics in $B_{d}^{0} \bar{B}_{d}^{0}$ mixing.
\end{abstract}

Flavor Physics \& CP Violation 2015

May 25-29, 2015

Nagoya, Japan

\footnotetext{
* Speaker.

${ }^{\dagger}$ This work is supported by the Norwegian research Council.
} 


\section{Introduction and Fit Methodology}

The phase of the Cabibbo-Kobayashi-Maskawa (CKM) matrix [1] produces $C P$ violation in the Standard Model (SM). Unitarity relations of the CKM matrix provide an excellent laboratory to test the SM predictions, in particular the relation $V_{u b}^{*} V_{u d}+V_{c b}^{*} V_{c d}+V_{t b}^{*} V_{t d}=0$ since many measurements in the $B$ and $K$ systems can be combined for this test.

The scan method is a frequentist-based technique for fitting elements of the CKM matrix [2], making no assumptions as to the distribution of theory errors. We account for theory uncertainties in the QCD parameters $f_{B_{s}}, f_{B_{s}} / f_{B_{d}}, B_{B_{s}}, B_{B_{s}} / B_{B_{d}}$, and $B_{K}$ and in the CKM parameters $\left|V_{u b}\right|$ and $\left|V_{c b}\right|$ by scanning over a plausible range of theory uncertainty using fixed grid or Monte Carlo (MC) methods. In the baseline fit, we combine measurements of $\Delta m_{d}, \Delta m_{s}, \varepsilon_{K},\left|V_{c b}\right|,\left|V_{u b}\right|,\left|V_{u d}\right|$, $\left|V_{u s}\right|, \sin 2 \beta, \alpha, \gamma, \mathscr{B}\left(B^{+} \rightarrow \tau^{+} v_{\tau}\right), \mathscr{B}\left(B_{s}^{0} \rightarrow \mu^{+} \mu^{-}\right)$and $\mathscr{B}\left(B_{s}^{0} \rightarrow \mu^{+} \mu^{-}\right)$in the $\chi^{2}$ function:

$$
\begin{aligned}
\chi^{2}\left(\bar{\rho}, \bar{\eta}, p_{i}, t_{j}\right) & =\left(\frac{\left\langle\Delta m_{d, s}\right\rangle-\Delta m_{d, s}\left(\bar{\rho}, \bar{\eta}, p_{i}, t_{j}\right)}{\sigma_{\Delta m_{d, s}}}\right)^{2}+\left(\frac{\left\langle\mid V_{c b, u b, u d, u s}\right\rangle-V_{c b, u b, u d, u s}\left(\bar{\rho}, \bar{\eta}, p_{i}, t_{j}\right)}{\sigma_{V_{c b}, u b, u d, u s}}\right)^{2} \\
& +\left(\frac{\left\langle\left|\varepsilon_{K}\right|\right\rangle-\varepsilon_{K}\left(\bar{\rho}, \bar{\eta}, p_{i}, t_{j}\right)}{\sigma_{\varepsilon_{K}}}\right)^{2}+\left(\frac{\left\langle S_{\psi K}\right\rangle-\sin 2 \beta\left(\bar{\rho}, \bar{\eta}, p_{i}\right)}{\sigma_{S_{S K}}}\right)^{2}+\left(\frac{\langle\alpha\rangle-\alpha\left(\bar{\rho}, \bar{\eta}, p_{i}\right)}{\sigma_{\alpha}}\right)^{2} \\
& +\left(\frac{\langle\gamma\rangle-\gamma\left(\bar{\rho}, \bar{\eta}, p_{i}\right)}{\sigma_{\gamma}}\right)^{2}+\sum_{k}\left(\frac{\left\langle\mathscr{M}_{k}\right\rangle-\mathscr{M}_{k}\left(p_{i}\right)}{\sigma_{\mathscr{M}_{k}}}\right)^{2}+\sum_{n}\left(\frac{\left\langle\mathscr{T}_{n}\right\rangle-\mathscr{T}_{n}\left(p_{i}, t_{j}\right)}{\sigma_{\mathscr{T}_{n}}}\right)^{2},
\end{aligned}
$$

where $p_{i}$ are measured quantities, including the Wolfenstein parameters $A$ and $\lambda$; the $t_{j}$ are QCD parameters. Errors on the $t_{j}$ are split into a "statistical" and a theory error. We account for the "statistical" error by including terms in the $\chi^{2}$ denoted by $\mathscr{T}_{n}$ in which the central values are taken from lattice calculations; we perform scans over the theory errors. To account for correlations among the observables, we add $\chi^{2}$ terms, denoted by $\mathscr{M}_{k}$, that include branching fractions, other CKM elements, Higgs, quark and $B$ meson masses, and $B$ meson lifetimes. Table 1 lists measured input values for baseline fits. Table 2 summarizes all the QCD parameters including $\eta_{c c}, \eta_{t c}, \eta_{t t}$ and $\eta_{b}$. We presently do not scan over the latter parameters, but account for them in the $\mathscr{T}_{n}$ terms. We parametrize $\eta_{c c}$ and its uncertainty in terms of $m_{c}$ and $\alpha_{s}$. For each choice of a set of theory parameters, we determine the $\chi^{2}$ and $P\left(\chi^{2} \geq \chi_{c}^{2} ; n-p+2 \mid H_{0}\right) \geq 0.05$ where $n(p)$ are the number of $\chi^{2}$ terms (number of fit parameters) and $H_{0}$ is the test hypothesis (e.g. the SM). For fits with $P\left(\chi^{2}\right)>5 \%$, we plot $\bar{\rho}-\bar{\eta}$ contours. The overlay of all contours present the $\bar{\rho}-\bar{\eta}$ region allowed in the SM. From the extrema of all displayed contours, we determine the range of UT parameters for a given confidence interval having made no assumption as to the distribution of theory errors.
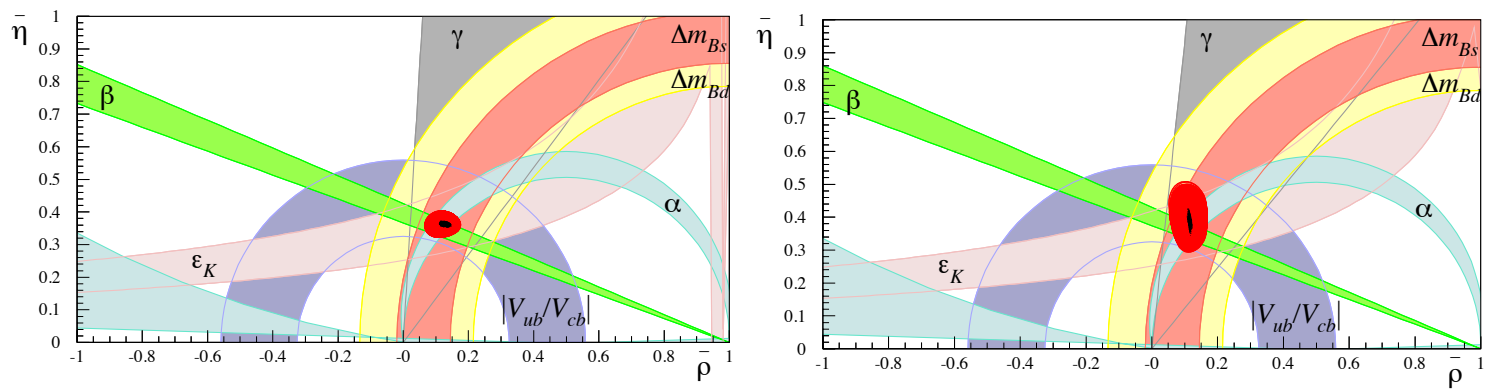

Figure 1: Overlay of $95 \%$ CL contours in the $\bar{\rho}-\bar{\eta}$ plane for the baseline fits without (left) and with NP parameters $r_{d}$ and $\theta_{d}$ (right). 
Table 1: Observables used in the baseline fits [3, 4].

\begin{tabular}{|c|c|c|c|}
\hline \hline$m_{t}\left[\mathrm{GeV} / \mathrm{c}^{2}\right]$ & $m_{c}\left[\mathrm{GeV} / \mathrm{c}^{2}\right]$ & $\Delta m_{d}\left[\mathrm{ps}^{-1}\right]$ & $\Delta m_{s}\left[\mathrm{ps}^{-1}\right]$ \\
\hline $173.2 \pm 0.87$ & $1.275 \pm 0.025$ & $0.510 \pm 0.003$ & $17.757 \pm 0.021$ \\
\hline \hline$\left|V_{c b}\right|$ & $\left|V_{u b}\right|$ & $\left|V_{u d}\right|$ & $\left|V_{u s}\right|$ \\
\hline \hline$(4.09 \pm 0.06 \pm 0.11) \times 10^{-2}$ & $(4.15 \pm 0.1 \pm 0.48) \times 10^{-3}$ & $0.97425 \pm 0.00022$ & $0.2252 \pm 0.0009$ \\
\hline$\left|V_{c d}\right|$ & $\left|V_{c s}\right|$ & $\left|V_{t b}\right|$ & \\
\hline \hline $0.23 \pm 0.11$ & $1.006 \pm 0.023$ & $0.97 \pm 0.08$ & \\
\hline$\varepsilon_{K}$ & $\sin 2 \beta$ & $\alpha$ & $\gamma$ \\
\hline \hline$(2.228 \pm 0.0011) \times 10^{-3}$ & $0.0691 \pm 0.017$ & $\left(85.1_{-2.0}^{+2.2}\right)^{o}$ & $\left(68.5_{-9.0}^{+7.9}\right)^{o}$ \\
\hline $\mathscr{B}\left(B^{+} \rightarrow \tau^{+} v\right)$ & $\mathscr{B}\left(B_{s}^{0} \rightarrow \mu^{+} \mu^{-}\right)$ & $\mathscr{B}\left(B_{d}^{0} \rightarrow \mu^{+} \mu^{-}\right)$ & \\
\hline \hline$(1.14 \pm 0.27) \times 10^{-4}$ & $\left(2.8_{-0.6}^{+0.7}\right) \times 10^{-9}$ & $\left(3.9_{-1.4}^{+1.6}\right) \times 10^{-10}$ & \\
\hline
\end{tabular}

Table 2: QCD parameters used in the fits with "statistical" and theory uncertainties, respectively [5].

\begin{tabular}{|l|c|c|c|c|}
\hline \hline$f_{B_{s}}[\mathrm{MeV}]$ & $f_{B_{s}} / f_{B_{d}}$ & $B_{B_{s}}$ & $B_{B_{s}} / B_{B_{d}}$ & $B_{K}$ \\
\hline $228.7 \pm 2.0 \pm 5.5$ & $1.311 \pm 0.046 \pm 0.076$ & $1.205 \pm 0.009 \pm 0.019$ & $1.053 \pm 0.04 \pm 0.064$ & $0.758 \pm 0.0020 \pm 0.019$ \\
\hline \hline$\eta_{c c}$ & $\eta_{t c}$ & $\eta_{t t}$ & $\eta_{b}$ & \\
\hline $1.39 \pm 0.35$ & $0.47 \pm 0.04$ & $0.5765 \pm 0.0065$ & $0.551 \pm 0.007$ & \\
\hline \hline
\end{tabular}

Table 3: The 95\% CL ranges from baseline fits without/with $\mathscr{B}\left(B_{s, d} \rightarrow \mu^{+} \mu^{-}\right)$for UT parameters.

\begin{tabular}{|l|c|c|c|c|c|}
\hline \hline Parameter & $\bar{\rho}$ & $\bar{\eta}$ & $\beta\left[^{o}\right]$ & $\alpha\left[^{o}\right]$ & $\gamma\left[^{o}\right]$ \\
\hline Baseline fit without $B_{s, d} \rightarrow \mu^{+} \mu^{-}$ & $0.077-0.176$ & $0.322-0.400$ & $19.9-24.9$ & $80.2-93.8$ & $63.6-77.8$ \\
Baseline fit with $B_{s, d} \rightarrow \mu^{+} \mu^{-}$ & $0.069-0.172$ & $0.324-0.401$ & $19.8-24.8$ & $79.0-93.3$ & $64.2-79.2$ \\
New physics fit with $B_{s, d} \rightarrow \mu^{+} \mu^{-}$ & $0.052-0.168$ & $0.294-0.509$ & $18.2-29.9$ & $70.0-95.1$ & $64.2-83.4$ \\
\hline \hline
\end{tabular}

\section{Results}

Figure 1 (left) shows the overlay of $\bar{\rho}-\bar{\eta}$ contours for all accepted baseline fits using 24 measurements ( $\left|V_{u d}\right|,\left|V_{u s}\right|,\left|V_{c b}\right|,\left|V_{c b}\right|,\left|V_{c d}\right|,\left|V_{c s}\right|,\left|V_{t b}\right|, \varepsilon_{K}, \Delta m_{B_{d}}, \Delta m_{B_{s}}, \sin 2 \beta, \alpha, \gamma, f_{B_{s}}, B_{B_{s}}$, $f_{B_{s} / f_{B_{d}}}, B_{B_{s}} / B_{B_{d}}, B_{K}, m_{t}, m_{c}, \mathscr{B}\left(B^{+} \rightarrow \tau^{+} v\right), \mathscr{B}\left(B_{s}^{0} \rightarrow \mu^{+} \mu^{-}\right)$and $\left.\mathscr{B}\left(B_{d}^{0} \rightarrow \mu^{+} \mu^{-}\right)\right)$to fit 12 parameters $\left(\bar{\rho}, \bar{\eta}, A, \lambda, f_{B_{s}}, B_{B_{s}}, f_{B_{s} / f_{B_{d}}}, B_{B_{s}} / B_{B_{d}}, B_{K}, m_{t}, m_{c}\right.$ and $\left.M_{H}\right)$. Table 3 shows the $95 \% \mathrm{CL}$ range of the unitarity triangle (UT) parameters for baseline fits without and with $\mathscr{B}\left(B_{s, d}^{0} \rightarrow \mu^{+} \mu^{-}\right)$.

New physics in $B_{d}^{0} \bar{B}_{d}^{0}$ mixing can be parameterized by a scale factor $r_{d}$ and a phase $\phi_{d}$ since

$$
\left\langle\bar{B}_{d}^{0}\left|\mathscr{H}^{N P}+\mathscr{H}^{S M}\right| B^{0}\right\rangle /\left\langle\bar{B}_{d}^{0}\left|\mathscr{H}^{S M}\right| B^{0}\right\rangle \equiv r_{d}^{2} \exp \left(2 i \theta_{d}\right)
$$

where $\mathscr{H}^{N P}\left(\mathscr{H}^{S M}\right)$ represent the new physics (SM) Hamiltonian. The scale factor modifies $\Delta m_{B_{d}}$ to $\left(\Delta m_{B_{d}}\right)^{N P}=r_{d}^{2}\left(\Delta m_{B_{d}}\right)^{S M}$ and $\left(\Delta m_{B_{s}} / \Delta m_{B_{d}}\right)^{N P}=\left(1 / r_{d}^{2}\right)\left(\Delta m_{B_{s}} / \Delta m_{B_{d}}\right)^{S M}$, and the phase modifies observables containing $\sin 2 \beta$ and $\sin 2 \alpha$ to $\sin 2\left(\beta+\theta_{d}\right)$ and $\sin 2\left(\alpha-\theta_{d}\right)$. To test this model, we add $r_{d}$ and $\theta_{d}$ to the baseline fit. Figure 1 (right) shows the resulting $\bar{\rho}-\bar{\eta}$ contours for all accepted baseline fits. The allowed region increases since the $\Delta m_{B_{d}}, \Delta m_{B_{s}} / \Delta m_{B_{d}}, \sin 2 \beta$ and $\sin 2 \alpha$ constraints are weakened due to compensation by the parameters $r_{d}$ and $\theta_{d}$. Figure 2 shows the $r_{d}-\theta_{d}$ contours that are consistent with the SM prediction. 


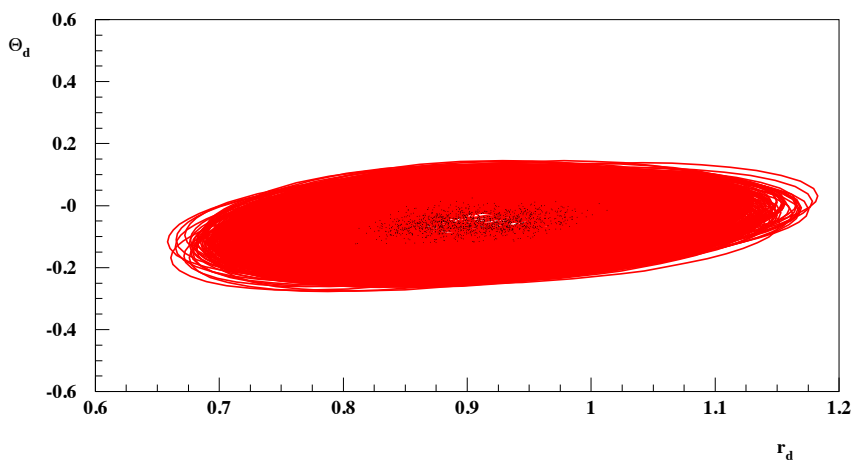

Figure 2: Overlay of $r_{d}-\theta_{d}$ contours. Black points show central values; the SM prediction is at $(1,0)$.

We further perform fits, called full fits, in which we replace $\alpha$ and $\gamma$ by measurements that determine them. These consist of all branching fractions and $C P$ asymmetries measured in $B \rightarrow P P, P V, V V$ modes and measurements of decay ratios and $C P$ asymmetries of $B^{+} \rightarrow D^{(*)} K^{+}$and $B^{+} \rightarrow D K^{*+}$, $B^{+} \rightarrow D^{(*)} \pi^{+}$and $B^{0} \rightarrow D^{(*)+} \rho^{-}$decays analyzed in the Giri-Grossman-Soffer-Zupan [6], GronauLondon-Wyler [7] and Atwood-Dunietz-Soni (ADS) methods [8]. Omitting $\mathscr{B}\left(B^{+} \rightarrow \tau^{+} v\right)$ and $\mathscr{B}\left(B_{s, d} \rightarrow \mu^{+} \mu^{-}\right)$, we fit to 230 measurements to determine 104 parameters. Following the Gronau-Rosner approach [9], we parametrize amplitudes in terms of tree, color-suppressed tree, penguin, singlet penguin, $W$-annihilation/ $W$-exchange and electroweak-penguin diagrams (up to $\lambda^{2}$ beyond leading order). In full fits, possible correlations between $\alpha(\gamma)$ and $\beta$ are accounted for, which is typically not the case in our baseline fits and fits by CKMfitter [10] and UTFIT [11].

We use the methodology of full fits to determine $\alpha$ and $\gamma$. We fit to 184 branching fractions and $C P$ asymmetry measurements in $B \rightarrow P P, B \rightarrow P V, B \rightarrow V V$ and $B \rightarrow a_{1} P$ modes to determine 95 parameters and extract $\alpha-\beta$ contours at $95 \% \mathrm{CL}$ shown in Fig. 3 (top). We fit to 61 rate ratio and $C P$ asymmetry measurements in $B^{ \pm} \rightarrow D^{(*) 0} K^{ \pm}, B^{ \pm} \rightarrow D^{0} K^{* \pm}, B^{ \pm} \rightarrow D^{(*) 0} \pi^{ \pm}$and $B^{ \pm} \rightarrow D^{0} \rho^{ \pm}$modes to determine 20 parameters and extract $\gamma-\beta$ contours at $95 \%$ CL shown in Fig. 3 (bottom) after scanning over $\left|V_{u b} / V_{c b}\right|$. Table 4 shows the fit results. Both $\alpha-\beta$ and $\gamma-\beta$ contours are consistent with the world average of $\beta=(21.9 \pm 0.7)^{\circ}$ from $B \rightarrow(c \bar{c}) K^{(*)}$ measurements [4]. Note that $\alpha$ and $\gamma$ are correlated with $\beta$ and that $\gamma$ depends on $\left|V_{u b} / V_{c b}\right|$.
Table 4: Measurements of $\alpha$ from fits to branching fractions and $C P$ asymmetries in $B \rightarrow P P, P V, V V, a_{1} P$ decays and measurements of $\gamma$ from fits to rate ratios and $C P$ asymmetries in $B^{ \pm} \rightarrow D^{(*) 0} K^{ \pm}, B^{ \pm} \rightarrow$ $D^{0} K^{* \pm}, B^{ \pm} \rightarrow D^{(*) 0} \pi^{ \pm}$and $B^{ \pm} \rightarrow D^{0} \rho^{ \pm}$ modes. The different $\gamma$ values result from the lowest/highest values of $\left|V_{u b} / V_{c b}\right|$. The magenta bar shows the world average for $\beta$ from $B \rightarrow c \bar{c} K^{(*)}$ modes.

\begin{tabular}{|l|c|c|c|}
\hline \hline Mode & $\alpha\left[^{o}\right]$ & $\beta\left[^{o}\right]$ & $\gamma\left[^{o}\right]$ \\
\hline$B \rightarrow P P, P V$, & & & \\
$V V, a_{1} P$ & $85.2_{-2.0}^{+2.1}$ & $21.7_{-1.6}^{+1.7}$ & \\
\hline$B \rightarrow D^{(*)} h$ & & $30.5_{-2.2}^{+2.3}$ & $67.7_{-9.2}^{+7.9}$ \\
& & $24.8_{-2.0}^{+2.0}$ & $69.1_{-8.8}^{+7.8}$ \\
\hline \hline
\end{tabular}

\section{3. "Wall" Plots}

We construct "Wall" plots to display the correlations among sets of three parameters. Orthogonal lines show the $\pm 1 \delta$ theory error range. The outer black contours result from probability 
requirements of $>32 \%$ or $>5 \%$. The inner black contours result from a $\pm 1 \delta$ requirement on all undisplayed variables. Colored solid contours result from a $\pm 1 \delta$ requirement on the out-of-plane variables. Colored dashed contours result from constraining the out-of-plane variables to their central values. Black dashed contours result from constraining all undisplayed variables to their central values. Figure 4 shows "Wall" plots for $\left|V_{u b}\right|, f_{B_{s}}$ and $B_{B_{S}}$ for $P\left(\chi^{2}\right)>32 \%$ and $P\left(\chi^{2}\right)>5 \%$.
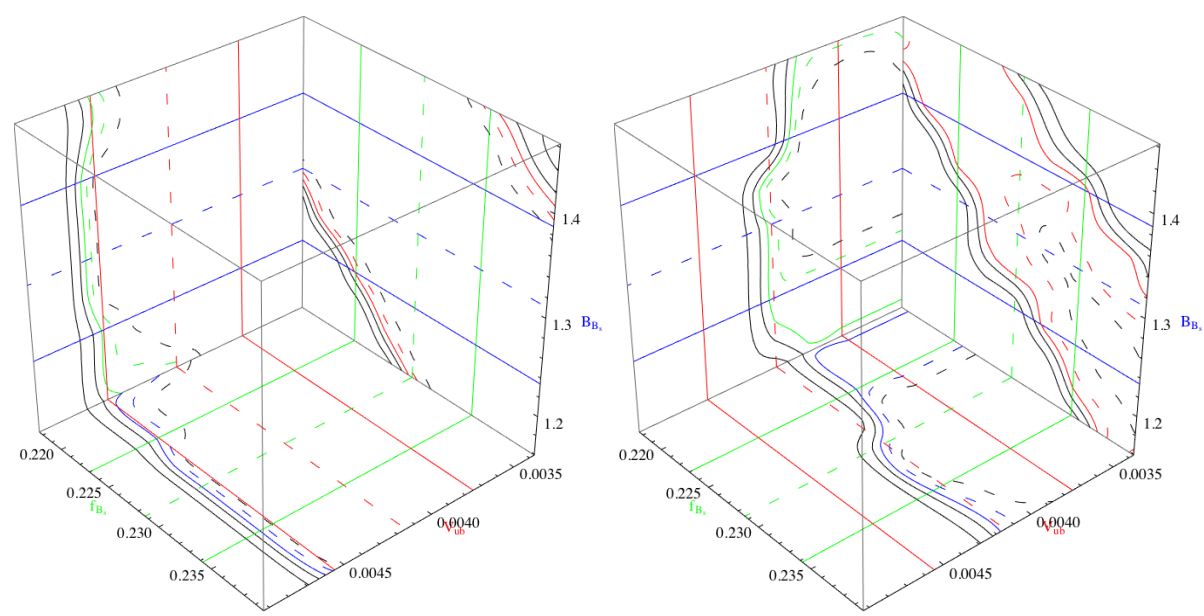

Figure 4: Wall plots for $\left|V_{u b}\right|, f_{B_{s}}$ and $B_{B_{S}}$ for $P\left(\chi^{2}\right)>5 \%$ (left) and $P\left(\chi^{2}\right)>32 \%$ (right).

\section{Conclusion}

Scan method baseline fits and full fits agree with the SM with no assumption as to the distribution of theory errors. The latter include correlations among $\alpha(\gamma)$ and $\beta$. Dedicated fits to $\alpha$ and $\gamma$ yield rather precise values. A new physics model in $B_{d}^{0} \bar{B}_{d}^{0}$ mixing increases the allowed $\bar{\rho}-\bar{\eta}$ region. "Wall" plots provide a means to determine whether any SM discrepancy originates from the values of theory parameters, experimental measurements or something else.

\section{References}

[1] N. Cabibbo, PRL 10, 531 (1963); M. Kobayashi, T. Maskawa, Prog.Th.Phys. 49, 652 (1973).

[2] G. Eigen et al., PRD 89033004 (2014).

[3] J. Beringer et al. (Particle Data Group), PR D86, 010001 (2012).

[4] D. Asner et al., arXiv:1010.1589v3 (2011); www.slac.stanford.edu/xorg/hfag/triangle/index.html

[5] J. Laiho et al., http://mypage.iu.edu/ elunghi/webpage/LatAves.

[6] A. Giri et al., PR D68, 054018 (2003).

[7] M. Gronau, D. London, PLB 253, 483 (1991); M. Gronau, D.Wyler, PLB 265, 172 (1991).

[8] D. Atwood, I. Dunietz, A. Soni, PRL 78, 3257 (1997); PRD 63, 036005 (2001).

[9] M. Gronau et al., PRD 50, 4520 (1994); PRD 60, 034021 (1999); PRD 61, 073008 (2000); PRD 62, 014031 (2000); PRD 69, 119901 (2004); A.S. Dighe et al., PRD 57, 1783 (1998); A. Beneke et al., PLB 638, 68 (2006).

[10] J. Charles et al., Eur. Phys. Jour. C41, 1 (2005); updates at http://ckmfitter.in2p3.fr/.

[11] M. Bona et al., JHEP 803, 049 (2008); updates at http://www.utfit.org/. 